\title{
Minds, motherboards, and money: futurism and realism in the neuroethics of $\mathrm{BCl}$ technologies
}

\author{
Mark A. Attiah ${ }^{1,2 *}$ and Martha J. Farah ${ }^{2,3}$ \\ ${ }^{1}$ Perelman School of Medicine, University of Pennsylvania, Philadelphia, PA, USA \\ ${ }^{2}$ Center for Neuroscience \& Society, University of Pennsylvania, Philadelphia, PA, USA \\ ${ }^{3}$ Department of Psychology, University of Pennsylvania, Philadelphia, PA, USA \\ *Correspondence: mattiah@mail.med.upenn.edu \\ Edited by: \\ Mikhail Lebedev, Duke University, USA \\ Reviewed by: \\ Manuel Casanova, University of Louisville, USA \\ Mikhail Lebedev, Duke University, USA
}

Keywords: neuroethics, brain computer interface (BCI), transhumanism, implants, experimental, enhancement

\section{INTRODUCTION}

Brain computer interfaces (BCIs) are systems that enable the brain to send and receive information to and from a computer, bypassing the body's own efferent and afferent pathways. BCIs have been used in experimental animal models to augment perception, motor control and even memory (Velliste et al., 2008; Berger et al., 2011; Torab et al., 2011). Human BCIs include cochlear implants and a host of experimental devices including retinal implants (Niparko et al., 2010; Klauke et al., 2011). BCI technology holds the potential to benefit humanity greatly, but also the potential to do harm, and its ethical implications have therefore been addressed by a number of commentators.

\section{THE PROBLEM WITH SCIENCE FICTION}

Commonly addressed ethical challenges with BCIs concern a future when the technology is used to give people superhuman abilities, or to control their thoughts and desires. The idea of human beings with computer-augmented brains engenders a wide range of reactions from laypersons, mostly negative (Lipsman et al., 2009; Lebedev, 2014). There is revulsion toward these so-called cyborgs occupying the "uncanny valley" between fellow human and robot (Mori, 1970). Some fear being overtaken by cyborgs with superior perceptual, cognitive and motoric powers while others are afraid that BCIs imposed upon us would result in mind control. Still others foresee tremendous benefit for humanity, with BCI enhancements elevating individuals and society.

Positive or negative, these images of computer-augmented brains are as speculative as they are vivid. Therein lie two significant challenges for ethical analysis of BCIs. First, we have strong emotional reactions to the prospects of humans with cyborg brains. Second, our limited real-world experience with BCIs deprives our ethical analyses of a foundation of pragmatic empirical knowledge. As a result, our perspectives on the ethical and societal impact of BCIs are based primarily on gut reactions, either "the wisdom of repugnance" (as exemplified by Francis Fukuyama's nomination of transhumanism as "The World's Most Dangerous Idea, 2004") or the blind faith that technology can fix and improve everything (as exemplified by the mission statement of Humanity+, which promotes "the ethical use of technology to expand human capacities... [to make them] better than well" (http:// humanityplus.org/).

In this essay, we describe an approach to the ethical analysis of BCIs that resists the emotional pull of futuristic scenarios without denying the importance of the questions they raise. In so doing, we address the more prosaic ethical issues that confront us now, which are too often overshadowed by the more sensational prospects of cyborgs, mind control and transhumanism. Finally, we indicate pathways through which seemingly fantastical futuristic scenarios can be more knowledgeably anticipated and addressed.

\section{THREE ERAS OF BCIS AND THEIR ETHICAL CHALLENGES}

As an organizing framework, we will distinguish among three different eras of BCI development, near-term, medium-term, and long-term, and distinguish among the different ethical issues associated with each.

\section{LONG-TERM CHALLENGES}

The futuristic ethical concerns already mentioned arise in the long-term future. We define this era as when BCIs are routinely used to augment human brains, giving users perceptual, cognitive and motoric abilities that may greatly exceed those of the unenhanced, and transforming emotional life as well. At this point, cyborgs will differ radically from unenhanced humans. One concern is that they will view us as a different, and inferior, life form, much as we now regard chimpanzees (Warwick, 2003). Loss of individuality is another long-term future scenario. If BCIs are used for direct brain-to-brain contact, this would enable new modes of communication but also lead to the possible replacement of individual mental lives by a network in which individual brains are mere nodes (Warwick, 2003).

Thoughts, feelings and memories that can be stored and shared as bits of data will not degrade as readily as human brain tissue. While other prosthetics such as artificial hips or heart valves can replace failing bodily structures, BCIs offer the first possibility of increased longevity of a person's consciousness. Immortality may even be possible (McGee and Maguire, 2007). 
These BCI scenarios lead to interesting questions about what it means to be human. Would we be human if we could see infrared light, memorize a phone book, or cause others to move by willing them to with our own mind? Would we be human if we ceded the role of smartest beings on earth to others, or would those enhanced beings be humanity's new form? Would we be human if our minds never operated independently from others'? If they operated for eternity? These questions raise the more general question of what it means to be human, and also the question of whether being human by any particular definition has intrinsic value. Although these questions are age-old, they arise with a new urgency in connection with advanced BCIs (McGee and Maguire, 2007).

BCIs could eventually bring about tectonic societal shifts. The effects of cochlear implants on deaf culture (Tucker, 1998) may presage the impact of BCIs on all aspects of human culture that are adapted to current human capabilities rather than the augmented, networked capabilities of future cyborgs. Global political trends such as the Arab Spring, attributed in part to social networking, could be distant harbingers of the effect of wide bandwidth BCIs that enable the sharing of thoughts and feelings among groups of individuals.

\section{MEDIUM-TERM CHALLENGES}

The ethical challenges of the medium term will be largely free of the existential and metaphysical dimensions just discussed, such as ceding dominion of the earth to others, merging our identities into a global network, or questioning the meaning and value of being human. Yet the emergence of these medium-term challenges will depend on BCI technology advancing significantly beyond its current state and therefore do still have a science fiction flavor. We take the defining feature of the medium-term to be the availability of BCI therapies for a wide range of human afflictions.

One set of ethical issues at this point will concern barriers to delivery of $\mathrm{BCI}$ therapies. One barrier is likely to be economic. The cost of BCIs in the coming decades is hard to predict, as is future societal support for universal access, but we can anticipate some degree of inequity in access to BCIs. Patient acceptance of implants, in particular the bioethicists' "yuck factor” (Niemelä, 2011) may also prevent or slow delivery of BCI therapies.

Another issue with a degree of bioethical precedent concerns control of the BCI (Jebari and Hansson, 2013). Therapies vary in how much control patients have. How much adjustment or programming of a BCI by the patient will be feasible, and with what risks and benefits? An aspect of control with much less precedent concerns the possibility of BCI hacking, that is, the possibility that a third party, unbeknownst to patient and healthcare provider, would deliver inputs to a patient's brain or read brain states (Denning et al., 2009). Although safeguards against such interventions could of course be designed into any system, experience with current information technology shows that no system can ever be made entirely invulnerable to such attacks. This raises the dire prospect of hacking into systems controlling human thought, feeling and behavior.

Finally, as BCIs are adopted more widely for therapeutic use, enhancement uses will likely follow. The ethics of enhancement is a well-explored area in bioethics and neuroethics more specifically (Parens, 2000; Farah et al., 2014). However, compared to the effects of drugs, which only modulate function within existing brain networks, the addition of new sensory, action and computational devices has the potential to more radically enhance human capabilities.

The transition from therapy to enhancement is evident in many areas of medicine: from neuropsychiatry, where attention-boosting drugs are used by college students to enhance their focus and improve their grades (Sussman et al., 2006), to plastic surgery, where techniques developed to reconstruct injured faces are now commonly used to enhance the attractiveness of the uninjured. Therapeutic implantation of BCIs for only mildly impaired individuals could provide a bridge between therapy and enhancement uses of BCI. Another pathway along which BCIs could transition from therapy to enhancement is by the addition of enhancing BCIs for someone who is already undergoing therapeutic implantation for a serious disorder, for example increasing the range of wavelengths perceived by someone with impaired vision.

\section{NEAR-TERM CHALLENGES}

Compared with the challenges awaiting us in the medium and long-term, which are currently speculative, we are now faced with a set of ethical challenges that are clear and present. How we respond to these challenges will determine the path we take to the medium- and long-term challenges of BCIs. One set of ethical challenges concerns clinical trials-how trials are conducted and which indications are pursued. Economic considerations of course shape these decisions, and the ethical issue is how these considerations will be balanced by other considerations. Will BCIs be developed only for the most profitable applications, leaving numerous "orphan" medical conditions unaddressed? How will an emphasis on profitability shape the future of BCI technology, by advancing technology for certain applications over others?

A related set of immediate challenges concerns ownership of products during research and development. The finances of pharmaceutical research and development has been identified as an impediment to discovery and innovation (Cohen, 2005), as well as a disincentive for scientific objectivity and transparency (Goldacre, 2013). Given the extensive collaboration of academic and other publicly funded researchers with privately held device manufacturers, how should we design intellectual property agreements, clinical trials and regulation to maximize innovation and patient benefit?

\section{APPROACHING THE FUTURE STEP BY STEP}

We cannot help but approach the future step by step. In contrast, ethical analyses sometimes jump ahead. This is motivated by the commendable desire to anticipate, and therefore more effectively address, the ethical issues of the future. But this long-term ethical forecasting can be counterproductive. One reason is that it takes our attention away from the vitally important, if more mundane, ethical issues of today. Pondering what it means to be human may be more interesting than projecting research and development 
Table 1 | Three eras of $\mathrm{BCls}$ and their corresponding ethical challenges.

\begin{tabular}{lll}
\hline Time period & Characteristics & Ethical challenges \\
\hline Long-term & $\begin{array}{l}\text { Extensive enhancement of } \\
\text { human brains with } \mathrm{BCls}\end{array}$ & $\begin{array}{l}\text { Transhuman cyborgs' treatment of humans as } \\
\text { inferior and inconsequential }\end{array}$
\end{tabular}

Vulnerability of those with $\mathrm{BCls}$ to thought control and mind-reading

Loss of individuality to a merged group mind

Immortality of thoughts, memories and whole minds

Medium-term Routine use of $\mathrm{BCls}$ as therapy Cost of $\mathrm{BCls}$ as an obstacle for needy patients

Discomfort or disgust with implants as an obstacle for patients

Question of who controls operation of patients'

$\mathrm{BCls}$

Security of $\mathrm{BCl}$ against hacking

Acceptance of $\mathrm{BCls}$ for enhancement of normal

function

Near-term Use of $\mathrm{BCls}$ in a translational research setting

\section{Conduct of clinical trials}

Developing $\mathrm{BCl}$ systems that maximize benefit as opposed to profit

Ownership of intellectual property as an impediment or incentive to biomedical advances

The influence of funding sources on research priorities

output under different intellectual property regimes. However, there is only one opportunity to get current decisions right, and that is now. Another reason is that bioethical decisions depend not only on values and principles, but also on the facts of the matter, which we have limited ability to anticipate in the distant future. Only as we live through each of the eras summarized in Table $\mathbf{1}$ will we be positioned to predict the likely empirical constraints on the ethical decisions of the next era.

For example, how current BCI work is financed, who controls intellectual property and so forth, will set our course toward the next set of issues, the decisions on what gets commercially developed, how patients get access, and what kind of enhancements are available. Then, depending on our experience with whatever mix of technologies is developed in that medium-term period-which sensory, motor and cognitive systems can be effectively augmented for therapy and enhancement, what unanticipated benefits and harms emerge-we will have a much better platform from which to foresee and address the currently imponderable long-term ethical challenges of BCIs and transhumanism.

\section{REFERENCES}

Berger, T. W., Hampson, R. E., Song, D., Goonawardena, A., Marmarelis, V. Z., and Deadwyler, S. A. (2011). A cortical neural prosthesis for restoring and enhancing memory. J. Neural. Eng. 8:046017. doi: 10.1088/1741-2560/ $8 / 4 / 046017$

Cohen, F. J. (2005). Macro trends in pharmaceutical innovation. Nat. Rev. Drug. Discov. 4, 78-84. doi: $10.1038 / \operatorname{nrd} 1610$

Denning, T., Matsuoka, Y., and Kohno, T. (2009). Neurosecurity: security and privacy for neural devices. Neurosurg. Focus 27:E7. doi: 10.3171/2009. 4.FOCUS0985

Farah, M. J., Smith, M. E., Ilieva, I., and Hamilton, R. H. (2014). Cognitive enhancement. Wiley Int. Rev. Cogn. Sci. 5, 95-103. doi: 10.1002/wcs. 1250

Goldacre, B. (2013). Bad Pharma: How Drug Companies Mislead Doctors and Harm Patients. New York, NY: Random House LLC.

Jebari, K., and Hansson, S.-O. (2013). European public deliberation on brain machine interface technology: five convergence seminars. Sci. Eng. Ethics 19, 1071-1086. doi: 10.1007/s11948-0129425-0

Klauke, S., Goertz, M., Rein, S., Hoehl, D., Thomas, U., Eckhorn, R., et al. (2011). Stimulation with a wireless intraocular epiretinal implant elicits visual percepts in blind humans. Invest. Ophthalmol. Vis. Sci. 52, 449-455. doi: 10.1167/ iovs. $09-4410$
Lebedev, M. (2014). Brain-machine interfaces: an overview. Trans. Neurosci. 5, 99-110. doi: 10.2478/ s13380-014-0212-z

Lipsman, N., Zener, R., and Bernstein, M. (2009). Personal identity, enhancement and neurosurgery: a qualitative study in applied neuroethics. Bioethics 23, 375-383. doi: 10.1111/j.1467-8519. 2009.01729.x

McGee, E. M., and Maguire, G. Q. (2007). Becoming borg to become immortal: regulating brain implant technologies. Camb. Q. Healthc. Ethics 16, 291-302. doi: 10.1017/S0963180107070326

Mori, M. (1970). The uncanny valley. Energy 7, 33-35. Niemelä, J. (2011). What Puts the 'Yuck' in the Yuck Factor?. Bioethics 25, 267-279. doi: 10.1111/j.14678519.2010.01802.x

Niparko, J. K., Tobey, E. A., Thal, D. J., Eisenberg, L. S., Wang, N.-Y., Quittner, A. L., et al. (2010). Spoken language development in children following cochlear implantation. JAMA 303, 1498-1506. doi: 10.1001/jama.2010.451

Parens, E. (Ed.). (2000). Enhancing Human Traits: Ethical and Social Implications. Washington, DC: Georgetown University Press.

Sussman, S., Pentz, M. A., Spruijt-Metz, D., and Miller, T. (2006). Misuse of "study drugs:" prevalence, consequences, and implications for policy. Subst. Abuse Treat. Prev. Policy 1:15. doi: 10.1186/ 1747-597X-1-15

Torab, K., Davis, T. S., Warren, D. J., House, P. A., Normann, R. A., and Greger, B. (2011). Multiple factors may influence the performance of a visual prosthesis based on intracortical microstimulation: nonhuman primate behavioural experimentation. J. Neural. Eng. 8:035001. doi: 10.1088/17412560/8/3/035001

Tucker, B. P. (1998). Deaf culture, cochlear implants, and elective disability. Hast. Cent. Rep. 28:6. doi: $10.2307 / 3528607$

Velliste, M., Perel, S., Spalding, M. C., Whitford, A. S., and Schwartz, A. B. (2008). Cortical control of a prosthetic arm for self-feeding. Nature 453, 1098-1101. doi: 10.1038/nature06996

Warwick, K. (2003). Cyborg morals, cyborg values, cyborg ethics - Springer. Ethic. Inf. Technol. 5, 131-137.

Conflict of Interest Statement: The authors declare that the research was conducted in the absence of any commercial or financial relationships that could be construed as a potential conflict of interest.

Received: 21 April 2014; accepted: 24 April 2014; published online: 15 May 2014.

Citation: Attiah MA and Farah MJ (2014) Minds, motherboards, and money: futurism and realism in the neuroethics of BCI technologies. Front. Syst. Neurosci. 8:86. doi: 10.3389/fnsys.2014.00086

This article was submitted to the journal Frontiers in Systems Neuroscience.

Copyright (c) 2014 Attiah and Farah. This is an openaccess article distributed under the terms of the Creative Commons Attribution License (CC BY). The use, distribution or reproduction in other forums is permitted, provided the original author(s) or licensor are credited and that the original publication in this journal is cited, in accordance with accepted academic practice. No use, distribution or reproduction is permitted which does not comply with these terms. 